\title{
An Atom-economic and Facile Synthesis of Novel 4-Imino-3-phenyl-2- substitutedphenyl-5-tolyl-2H,3H,5H[1,2,5]thiadiazolidine-1-oxide through 1,3-Dipolar Cycloaddition Reactions
}

Vasudha Kaushal, Anjandeep Kaur* and Baldev Singh

Department of Chemistry, Punjabi University, Patiala, Punjab, India

\begin{abstract}
An atom economic and facile synthesis of novel thiadiazolidine-1-oxides has been achieved via using 1,3-dipolar cycloaddition reactions. The salient features of synthetic procedure are characterized by the good yields, high regio- and stereoselectivity, one-pot procedure, and operational simplicity. The regiochemistry and structures of the cycloadducts were determined by using various spectroscopic techniques (IR, ${ }^{1} \mathrm{H}-\mathrm{NMR}, \mathrm{ESI}-\mathrm{MS}$ ) and elemental analyses data.
\end{abstract}

Keywords: Atom-economic; Thiadiazolidine; Stereoselectivity; Antiparasitic; Antiviral; Cycloaddition

\section{Introduction}

Organic synthesis has been one of the most successful scientific disciplines, and has also been of enormous practical utility. In the course of few last years, the progress of organic synthesis has been manifold and has gained importance in the field of heterocyclic compounds. This synthetic organic chemistry provides cornucopia of heterocyclic systems. Among various synthetic methods, cycloaddition reactions involving two simple components appear to be an attractive choice for the stereoselective synthesis of heterocyclic compounds due to its atom-economic and facile nature. Compounds incorporating heterocyclic ring systems continue to attract considerable interest due to the wide range of biological activities they possess. Amongst them, five-membered heterocyclic compounds occupy a unique place in the realm of natural and synthetic organic chemistry. Five-membered heterocycles like thiadiazolidine have found wide applications in the fields of pharmaceutical chemistry and have stimulated much interest in the field of medicinal and biological chemistry. The value of thiadiazolidine derivatives is significant among various heterocycles, as they are found to possess antibacterial [1-4], anti-inflammatory [5,6], antiviral [7], antiparasitic [8], antifungal [9-11] and other diverse biological activities [12]. Many thiadiazolidines are used for the production of anticonvulsant drugs $[13,14]$ and in the treatment of depression also [15]. In addition to this, thiadiazolidine derivatives have played a crucial role in the theoretical development of heterocyclic chemistry and are also used extensively in organic synthesis.

Encouraged by the diverse biological activities of thiadiazolidine substituted compounds, in our investigation we found an interesting approach to synthesize these substituted ring systems.

\section{Experimental}

\section{General}

Unless otherwise indicated, all common reagents were used as obtained from commercial suppliers (Sigma Aldrich) without further purification and the solvents were dried before use. All melting points were recorded on Gallen-Kamp apparatus and are uncorrected. IR spectra were recorded on a Perkin Elmer RXIFT infrared spectrophotometer (manufactured at Buckinghamshire, England) using $\mathrm{KBr}$ pellets. ${ }^{1} \mathrm{H}-\mathrm{NMR}$, were recorded at $400 \mathrm{MHz}$ on BRUKER spectrometer (manufactured at Fallanden, Switzerland) using tetramethylsilane (TMS) as internal standard. Mass spectra were recorded on Waters Micromass Q-T of Micro (ESI) spectrometer (manufactured at Vernon Hills, USA). Elemental analysis was carried out using Elementar Vario MICRO cube CHN analyzer (Frankfurt, Germany). Thin-layer chromatography (TLC) analysis was carried out on glass plates coated with silica gel-G (Loba Chemie) suspended in methanol-chloroform. Column chromatography was performed using silica gel (60-120 mesh, Loba Chemie).

\section{General procedure for the synthesis of substituted} benzalaniline ( $3 a-i)$

The solution of benzaldehyde $(0.01 \mathrm{~mol})$ in ethanol $(15 \mathrm{~mL})$ taken in $100 \mathrm{~mL}$ beaker was added to the solution of substituted aniline $(0.01$ $\mathrm{mol})$ in ethanol $(15 \mathrm{~mL})$ at room temperature. The reaction mixture was stirred for half an hour and then cooled in an ice bath for fifteen minutes (Scheme 1). The crude compound separated out was filtered at the suction pump and recrystallised from ethanol.

General procedure for the synthesis of $N$ - $\alpha$-cyano- $\alpha$-phenyl methylaniline (4a-i)

To substituted benzalaniline $(0.02 \mathrm{~mol})$ taken in $250 \mathrm{~mL}$ conical

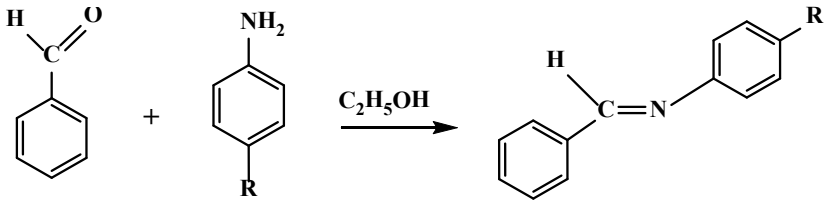

(1)
(2)
(3)

$$
\begin{array}{lll}
\text { 2a, 3a: } \mathrm{R}=-\mathrm{H} & \mathbf{2 d}, \mathbf{3 d}: \mathrm{R}=-\mathrm{Cl} & \mathbf{2 g}, \mathbf{3} \mathbf{g}: \mathrm{R}=-\mathrm{OH} \\
\text { 2b, 3b: } \mathrm{R}=-\mathrm{CH}_{3} & \mathbf{2 e}, \mathbf{3 e}: \mathrm{R}=-\mathrm{NO}_{2} & \mathbf{2 h}, \mathbf{3 h}: \mathrm{R}=-\mathrm{F} \\
\mathbf{2 c}, \mathbf{3 c}: \mathrm{R}=-\mathrm{OCH}_{3} & \mathbf{2 f}, \mathbf{3 f}: \mathrm{R}=-\mathrm{Br} & \mathbf{2 i}, \mathbf{3 i}: \mathrm{R}=-\mathrm{N}\left(\mathrm{CH}_{3}\right)_{2}
\end{array}
$$

Scheme 1: Schematic diagram describing the synthesis of variously substituted Azomethines.

*Corresponding author: Anjandeep Kaur, Department of Chemistry, Punjabi University, Patiala-147 002, Punjab, India, Tel: +91-9872666431; E-mail kauranjandeep@gmail.com

Received November 06, 2015; Accepted November 20, 2015; Published November 25, 2015

Citation: Kaushal V, Kaur A, Singh B (2015) An Atom-economic and Facile Synthesis of Novel 4-Imino-3-phenyl-2-substitutedphenyl-5-tolyl-2H,3H,5H[1,2,5] thiadiazolidine-1-oxide through 1,3-Dipolar Cycloaddition Reactions. Med chem 5 484-488. doi: 10.4172/2161-0444.1000306

Copyright: ( $) 2015$ Kaushal V, et al. This is an open-access article distributed under the terms of the Creative Commons Attribution License, which permits unrestricted use, distribution, and reproduction in any medium, provided the original author and source are credited. 
Citation: Kaushal V, Kaur A, Singh B (2015) An Atom-economic and Facile Synthesis of Novel 4-Imino-3-phenyl-2-substitutedphenyl-5-tolyl$2 H, 3 H, 5 H[1,2,5]$ thiadiazolidine-1-oxide through 1,3-Dipolar Cycloaddition Reactions. Med chem 5: 484-488. doi: 10.4172/21610444.1000306

flask were added ethanol $(50 \mathrm{~mL})$ and glacial acetic acid $(5-6 \mathrm{~mL})$. To this solution was added a solution of aqueous potassium cyanide $(0.01$ mol) in distilled water $(10 \mathrm{~mL})$ in small installments. The reaction mixture was stirred for one hour and then allowed to stay overnight (Scheme 2). On dilution with distilled water the crude product was separated out. The product was filtered, washed with distilled water and recrystallised from petroleum ether to give crystalline $N$ - $\alpha$-cyano- $\alpha$ phenylmethylaniline.

\section{General procedure for the synthesis of $N$-sulphinyl-4-toluidine (7)}

A solution of pure thionyl chloride $(0.69 \mathrm{~mol})$ in $100 \mathrm{~mL}$ of anhydrous toluene was added slowly to a solution of recrystallised 4-toluidine in $250 \mathrm{~mL}$ of anhydrous benzene contained in a 1-litre capacity round bottomed flask with swirling motion and occasional cooling in icebath as the reaction was an exothermic one. Each successive addition of thionyl chloride solution was done only after the previous reaction had subsided. An immediate precipitation of toluidinum sulphinyl chloride occurs. After the addition of the thionyl chloride solution was complete, the mixture was heated to reflux using a calcium chloride guard tube on a heating mantle until a clear solution was obtained. Whole of the solid disappeared in about five hours and the reaction mixture was refluxed for another hour to complete the reaction (Scheme 3). The solvent and the excess of thionyl chloride were distilled off under reduced pressure to yield a yellow $N$-sulphinyl-4-toluidine.

General procedure for the synthesis of 4-imino-3-phenyl-2substitutedphenyl-5-tolyl- $2 H, 3 H, 5 H[1,2,5]$ thiadiazolidine1-oxide (8a-i)

To the solution of $N$ - $\alpha$-cyano- $\alpha$-phenylmethylaniline $(0.01 \mathrm{~mol})$ in anhydrous toluene taken in a $100 \mathrm{~mL}$ round bottomed flask were added few drops of triethylamine and $N$-sulphinyl-4-toluidine $(0.01 \mathrm{~mol})$ at room temperature. The reaction mixture after refluxing for $30-40$ minutes was allowed to stand for overnight period. The reaction mixture on dilution with solvent ether gave the crude product (Scheme 4) which was subjected to column chromatography using hexane:ethyl acetate

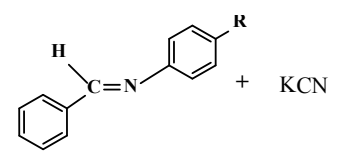

(3)

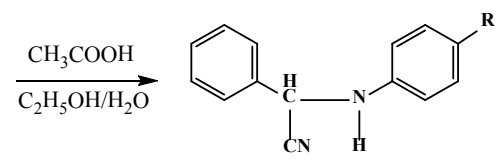

(4)

$3 \mathbf{3}, \mathbf{4}_{\mathbf{g}}: \mathrm{R}=-\mathrm{OH}$ 3i, $4 \mathrm{i}: \mathrm{R}=-\mathrm{N}\left(\mathrm{CH}_{3}\right)_{2}$
Scheme 2: Schematic diagram describing the synthesis of substituted $N$ - $\alpha$ cyanoamines.

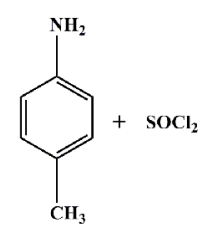

(5)

(6)

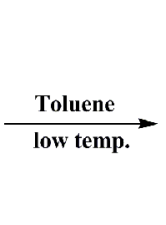

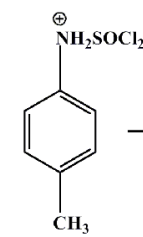

$\mathrm{CH}_{3}$

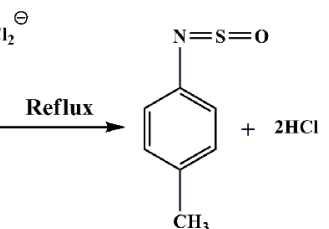

(7)
Scheme 3: Schematic diagram describing the synthesis of $\mathrm{N}$-sulphinyl-4toluidine.
(8:2) as an eluent to afford pure 4-imino-3-phenyl-2-substitutedphenyl5-tolyl-2H,3H,5H[1,2,5]thiadiazolidin-1-oxide derivatives $8 \mathrm{a}$-i.

4-Imino-2-(4'-tolyl)-5-tolyl-3-phenyl- $2 H, 3 H, 5 H[1,2,5]$ thiadiazolidine-1-oxide (8a): Compound obtained as yellow solid; $68 \%$ yield; m.p. $116-118^{\circ} \mathrm{C}$; IR ( $\mathrm{KBr}$ pellets): $3329 \mathrm{~cm}^{-1}(\mathrm{~N}-\mathrm{H}) ; 1616$ $\mathrm{cm}^{-1}(\mathrm{C}=\mathrm{N}) ; 1590 \mathrm{~cm}^{-1}(\mathrm{C}=\mathrm{C}) ; 1029 \mathrm{~cm}^{-1}(\mathrm{~S}=\mathrm{O}) ;{ }^{1} \mathrm{H}-\mathrm{NMR}(400 \mathrm{MHz}$, $\left.\mathrm{CDCl}_{3}\right), \delta 6.64-8.06(\mathrm{~m}, 14 \mathrm{H}) ; \delta 5.31(\mathrm{~s}, 1 \mathrm{H}) ; \delta 2.33(\mathrm{~s}, 3 \mathrm{H}) ; \delta 2.30(\mathrm{~s}$, $3 \mathrm{H}$ ); MS(ESI): m/z 375[M+ $\mathrm{M}^{+}$; Anal. Calc. for $\mathrm{C}_{22} \mathrm{H}_{21} \mathrm{~N}_{3} \mathrm{OS}$ : C, 70.39; $\mathrm{H}$, 5.59; N, 11.19; S, 8.52, Found: C, 70.42; H, 5.60; N, 11.20; S, 8.53.

2,3-Diphenyl-4-imino-5-tolyl-2H,3H,5H[1,2,5] thiadiazolidine1-oxide (8b): Compound obtained as yellow solid; 64\% yield; m.p. 122$124^{\circ} \mathrm{C}$; IR (KBr pellets): $3332 \mathrm{~cm}^{-1}(\mathrm{~N}-\mathrm{H}) ; 1622 \mathrm{~cm}^{-1}(\mathrm{C}=\mathrm{N}) ; 1595 \mathrm{~cm}^{-1}$ $(\mathrm{C}=\mathrm{C}) ; 1024 \mathrm{~cm}^{-1}(\mathrm{~S}=\mathrm{O}) ;{ }^{1} \mathrm{H}-\mathrm{NMR}\left(400 \mathrm{MHz}, \mathrm{CDCl}_{3}\right), \delta 6.62-8.03(\mathrm{~m}$, $14 \mathrm{H}) ; \delta 5.30$ (s, $1 \mathrm{H}) ; \delta 2.30$ (s, $3 \mathrm{H}) ; \mathrm{MS}(\mathrm{ESI}): \mathrm{m} / \mathrm{z} 361\left[\mathrm{M}^{+}\right]$; Anal. Calc. for $\mathrm{C}_{21} \mathrm{H}_{19} \mathrm{~N}_{3} \mathrm{OS}$ : C, 69.80; H, 5.26; N, 11.63; S, 8.85, Found: C, 69.72; H, $5.25 ; \mathrm{N}, 11.57 ; \mathrm{S}, 8.86$.

4 - Imino- 2 - ( 4 ' - methoxyphenyl) - 5 - tolyl-3 - phenyl$2 H, 3 H, 5 H[1,2,5]$ thiadiazolidine-1-oxide (8c): Compound obtained as light yellow; $62 \%$ yield; m.p. $120-122^{\circ} \mathrm{C}$; IR ( $\mathrm{KBr}$ pellets): $3330 \mathrm{~cm}^{-1}$ $(\mathrm{N}-\mathrm{H}) ; 1620 \mathrm{~cm}^{-1}(\mathrm{C}=\mathrm{N}) ; 1588 \mathrm{~cm}^{-1}(\mathrm{C}=\mathrm{C}) ; 1030 \mathrm{~cm}^{-1}(\mathrm{~S}=\mathrm{O}) ;{ }^{1} \mathrm{H}-\mathrm{NMR}$ $\left(400 \mathrm{MHz}, \mathrm{CDCl}_{3}\right), \delta 6.70-8.10(\mathrm{~m}, 14 \mathrm{H}) ; \delta 5.40(\mathrm{~s}, 1 \mathrm{H}) ; \delta 3.67(\mathrm{~s}, 3 \mathrm{H})$; $\delta 2.34$ (s, 3H); MS(ESI): m/z 391[ $\left.\mathrm{M}^{+}\right]$; Anal. Calc. for $\mathrm{C}_{22} \mathrm{H}_{21} \mathrm{~N}_{3} \mathrm{O}_{2} \mathrm{~S}: \mathrm{C}$, 67.51; H, 5.37; N, 10.74; S, 8.17, Found: C, 67.48; H, 5.36; N, 10.73; S, 8.18 .

2-(4'-Chlorophenyl)-4-imino-5-tolyl-3-phenyl-2H,3H,5H[1,2,5] thiadiazolidine-1-oxide (8d): Compound obtained as light yellow solid; $70 \%$ yield; m.p. $102-104^{\circ} \mathrm{C}$; IR (KBr pellets): $3325 \mathrm{~cm}^{-1}(\mathrm{~N}-\mathrm{H})$; $1618 \mathrm{~cm}^{-1}(\mathrm{C}=\mathrm{N}) ; 1595 \mathrm{~cm}^{-1}(\mathrm{C}=\mathrm{C}) ; 1026 \mathrm{~cm}^{-1}(\mathrm{~S}=\mathrm{O}) ;{ }^{1} \mathrm{H}-\mathrm{NMR}(400$ $\left.\mathrm{MHz}, \mathrm{CDCl}_{3}\right), \delta 6.44-8.03(\mathrm{~m}, 14 \mathrm{H}) ; \delta 5.33(\mathrm{~s}, 1 \mathrm{H}) ; \delta 2.33(\mathrm{~s}, 3 \mathrm{H})$; MS(ESI): m/z $395\left[\mathrm{M}^{+}\right], 397\left[\mathrm{M}^{+}+2\right]$; Anal. Calc. for $\mathrm{C}_{21} \mathrm{H}_{18} \mathrm{ClN}_{3} \mathrm{OS}: \mathrm{C}$, 63.71; H, 4.55; N, 10.61; S, 8.08, Found: C, 63.69; H, 4.54; N, 10.60; S, 8.09 .

4-Imino-5-tolyl-2-(4'-nitrophenyl)-3-phenyl-2H,3H,5H[1,2,5] thiadiazolidine-1-oxide (8e): Compound obtained as yellow solid, $65 \%$ yield, m.p. $154-156^{\circ} \mathrm{C}$; IR ( $\mathrm{KBr}$ pellets): $3330 \mathrm{~cm}^{-1}(\mathrm{~N}-\mathrm{H}) ; 1625$ $\mathrm{cm}^{-1}(\mathrm{C}=\mathrm{N}) ; 1587 \mathrm{~cm}^{-1}(\mathrm{C}=\mathrm{C}) ; 1029 \mathrm{~cm}^{-1}(\mathrm{~S}=\mathrm{O}) ;{ }^{1} \mathrm{H}-\mathrm{NMR}(400 \mathrm{MHz}$ $\left.\mathrm{CDCl}_{3}\right), \delta 6.52-8.03(\mathrm{~m}, 14 \mathrm{H}) ; \delta 5.36(\mathrm{~s}, 1 \mathrm{H}) ; \delta 2.36$ (s, 3H); MS(ESI): $\mathrm{m} / \mathrm{z}$ 406[M+ $\mathrm{M}^{+}$; Anal. Calc. for $\mathrm{C}_{21} \mathrm{H}_{18} \mathrm{~N}_{4} \mathrm{O}_{3} \mathrm{~S}$ : C, 62.06; H, 4.43; N, 13.79; S, 7.87, Found: C, 62.08; H, 4.42; N, 13.78; S 7.88.

2-(4'-Bromophenyl)-4-imino-5-tolyl-3-phenyl-2H,3H,5H[1,2,5] thiadiazolidine-1-oxide (8f): Compound obtained as yellow solid; $72 \%$ yield, m.p. $114-116^{\circ} \mathrm{C}$; IR ( $\mathrm{KBr}$ pellets): $3320 \mathrm{~cm}^{-1}(\mathrm{~N}-\mathrm{H}) ; 1616$ $\mathrm{cm}^{-1}(\mathrm{C}=\mathrm{N}) ; 1592 \mathrm{~cm}^{-1}(\mathrm{C}=\mathrm{C}) ; 1024 \mathrm{~cm}^{-1}(\mathrm{~S}=\mathrm{O}) ;{ }^{1} \mathrm{H}-\mathrm{NMR}(400 \mathrm{MHz}$, $\left.\mathrm{CDCl}_{3}\right), \delta 6.64-8.04(\mathrm{~m}, 14 \mathrm{H}) ; \delta 5.31(\mathrm{~s}, 1 \mathrm{H}) ; \delta 2.41$ (s, 3H); MS(ESI): $\mathrm{m} / \mathrm{z} 440\left[\mathrm{M}^{+}\right], 442\left[\mathrm{M}^{+}+2\right]$; Anal. Calc. for $\mathrm{C}_{21} \mathrm{H}_{18} \mathrm{BrN}_{3} \mathrm{OS}: \mathrm{C}, 57.27 ; \mathrm{H}$, 4.09; N, 9.54; S, 7.26, Found: C, 57.25; H, 4.08; N, 9.53; S, 7.27.

2 - ( 4 ' - Hydroxy phenyl) - 4 - im in o- 5 - tolyl- 3 - phenyl$2 H, 3 H, 5 H[1,2,5]$ thiadiazolidine-1-oxide (8g): Compound obtained as light yellow solid; $74 \%$ yield; m.p. $118-120^{\circ} \mathrm{C}$; IR ( $\mathrm{KBr}$ pellets): 3326 $\mathrm{cm}^{-1}(\mathrm{~N}-\mathrm{H}) ; 1612 \mathrm{~cm}^{-1}(\mathrm{C}=\mathrm{N}) ; 1590 \mathrm{~cm}^{-1}(\mathrm{C}=\mathrm{C}) ; 1029 \mathrm{~cm}^{-1}(\mathrm{~S}=\mathrm{O})$; ${ }^{1} \mathrm{H}-\mathrm{NMR}\left(400 \mathrm{MHz}, \mathrm{CDCl}_{3}\right), \delta 8.09(\mathrm{~s}, 1 \mathrm{H}) ; 6.42-8.01(\mathrm{~m}, 14 \mathrm{H}) ; \delta$ 5.33 (s, 1H); $\delta 2.33$ (s, 3H); MS(ESI): m/z $377\left[\mathrm{M}^{+}\right]$; Anal. Calc. for $\mathrm{C}_{21} \mathrm{H}_{19} \mathrm{~N}_{3} \mathrm{O}_{2} \mathrm{~S}: \mathrm{C}, 66.84 ; \mathrm{H}, 5.04 ; \mathrm{N}, 11.14 ; \mathrm{S}, 8.48$, Found: C, 66.81; H, $5.03 ; \mathrm{N}, 11.12 ; \mathrm{S}, 8.49$.

2-(4'-Flourophenyl)-4-imino-5-tolyl-3-phenyl-2H,3H,5H[1,2,5] thiadiazolidine-1-oxide (8h): Compound obtained as light yellow solid; $69 \%$ yield; m.p. $126-128^{\circ} \mathrm{C}$; IR ( $\mathrm{KBr}$ pellets): $3329 \mathrm{~cm}^{-1}(\mathrm{~N}-\mathrm{H})$; $1620 \mathrm{~cm}^{-1}(\mathrm{C}=\mathrm{N}) ; 1598 \mathrm{~cm}^{-1}(\mathrm{C}=\mathrm{C}) ; 1023 \mathrm{~cm}^{-1}(\mathrm{~S}=\mathrm{O}) ;{ }^{1} \mathrm{H}-\mathrm{NMR}(400$ 
Citation: Kaushal V, Kaur A, Singh B (2015) An Atom-economic and Facile Synthesis of Novel 4-Imino-3-phenyl-2-substitutedphenyl-5-tolyl$2 H, 3 H, 5 H[1,2,5]$ thiadiazolidine-1-oxide through 1,3-Dipolar Cycloaddition Reactions. Med chem 5: 484-488. doi: 10.4172/21610444.1000306

$\left.\mathrm{MHz}, \mathrm{CDCl}_{3}\right), \delta 6.52-8.12(\mathrm{~m}, 14 \mathrm{H}) ; \delta 5.43(\mathrm{~s}, 1 \mathrm{H}) ; \delta 2.38(\mathrm{~s}, 3 \mathrm{H})$; MS(ESI): $\mathrm{m} / \mathrm{z} 378\left[\mathrm{M}^{+}\right]$; Anal. Calc. for $\mathrm{C}_{21} \mathrm{H}_{18} \mathrm{FN}_{3} \mathrm{OS}$ : C, 66.67; $\mathrm{H}, 4.76$; N, 11.11; S, 8.46, Found: C, 66.72; H, 4.74; N, 11.07; S, 8.42.

4-Imino-2-[4'-(dimethylamino)phenyl]-5-tolyl-3-phenyl$2 H, 3 H, 5 H[1,2,5]$ thiadiazolidine-1-oxide $(8 \mathrm{i})$ : Compound obtained as yellow solid; $72 \%$ yield; m.p. $122-124^{\circ} \mathrm{C}$; IR ( $\mathrm{KBr}$ pellets): $3332 \mathrm{~cm}^{-1}$ $(\mathrm{N}-\mathrm{H}) ; 1618 \mathrm{~cm}^{-1}(\mathrm{C}=\mathrm{N}) ; 1590 \mathrm{~cm}^{-1}(\mathrm{C}=\mathrm{C}) ; 1027 \mathrm{~cm}^{-1}(\mathrm{~S}=\mathrm{O}) ;{ }^{1} \mathrm{H}-\mathrm{NMR}$ $\left(400 \mathrm{MHz}, \mathrm{CDCl}_{3}\right), \delta 6.52-8.12(\mathrm{~m}, 14 \mathrm{H}) ; \delta 5.43(\mathrm{~s}, 1 \mathrm{H}) ; \delta 2.74(\mathrm{~s}, 3 \mathrm{H})$, $\delta 2.80$ (s, 3H); $\delta 2.38$ (s, 3H); MS(ESI): $\mathrm{m} / \mathrm{z} 404\left[\mathrm{M}^{+}\right]$; Anal. Calc. for $\mathrm{C}_{23} \mathrm{H}_{24} \mathrm{~N}_{4}$ OS: C, 68.31; H, 5.94; N, 13.86; S, 7.92, Found: C, 68.26; H, $5.91 ; \mathrm{N}, 13.82 ; \mathrm{S}, 7.94$.

\section{Results and Discussion}

The survey of literature reveals that very few amount of work of $N$-sulphinylanilines have been taken with $N$ - $\alpha$-cyanoamines [16]. The present work aims therefore to study the effect of substituent on aniline part and hence to study their behavior on these cycloaddition reactions and to fill the gap in the literature present study has been taken up in this direction. For the present study, dienophile with cumulative double bond $\mathrm{N}$-sulphinyl-4-toluidine has been used for these cycloaddition reactions. $N$-sulphinyl-4-toluidine has been synthesized from pure (AR) grade $p$-toluidine which was further purified by recrystallisation and using doubly distilled thionyl chloride as reported in literature [17]. The $\mathrm{N}$-sulphinyl-4-toluidine so obtained was dried over anhydrous sodium sulphate for an overnight period and this was used after distillation in vacuo for these cycloaddition reactions with the $N$ - $\alpha$-cyanoamines.

Variously substituted $N$ - $\alpha$-cyanoamines were synthesized by following identical procedure as reported in literature [16]. In the first step, variously substituted azomethines were synthesized by condensing substituted anilines with benzaldehyde in alcohol as solvent (Scheme 1) which were subsequently subjected to hydrocyanation using potassium cyanide in aqueous ethanolic solution containing glacial acetic acid and the usual work up yielded crude crystalline $N$ - $\alpha$-cyanoamines (Scheme 2; Table 1).

Then to the solution of $N$ - $\alpha$-cyanoamine in dry toluene a catalytic amount of triethylamine was added followed by the addition of $\mathrm{N}$-sulphinyl-4-toluidine 7 (which itself was synthesized using toluidine and thionyl chloride Scheme 3) to provide the desired five-membered heterocyclic compound (Scheme 4) which have been characterized as 4-imino-3-phenyl-2-substitutedphenyl-5-tolyl- $2 H, 3 H, 5 H[1,2,5]$

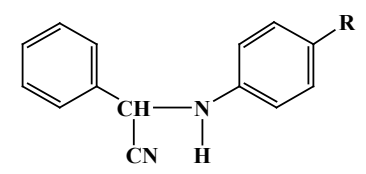

\begin{tabular}{|c|c|c|c|c|}
\hline Compounds & $\mathbf{R}$ & $\begin{array}{c}\text { Melting points } \\
\text { in }{ }^{\circ} \mathbf{C}\end{array}$ & $\begin{array}{c}\text { Colour of } \\
\text { compounds }\end{array}$ & \% Yield \\
\hline $\mathbf{4 a}$ & $-\mathrm{H}$ & $80-84$ & White & 65 \\
\hline $\mathbf{4 b}$ & $-\mathrm{CH}_{3}$ & $110-114$ & White & 70 \\
\hline $\mathbf{4 c}$ & $-\mathrm{OCH}_{3}$ & $44-48$ & Grey & 83 \\
\hline $\mathbf{4 d}$ & $-\mathrm{Cl}_{\mathbf{d}}$ & $78-80$ & White & 58 \\
\hline $\mathbf{4 e}$ & $-\mathrm{NO}_{2}$ & $140-144$ & Yellow & 81 \\
\hline $\mathbf{4 f}$ & $-\mathrm{Br}$ & $90-94$ & White & 68 \\
\hline $\mathbf{4 g}$ & $-\mathrm{OH}$ & $108-110$ & Yellow & 69 \\
\hline $\mathbf{4 h}$ & $-\mathrm{F}$ & $102-104$ & White & 67 \\
\hline $\mathbf{4 i}$ & $-\mathrm{N}\left(\mathrm{CH}_{3}\right)_{2}$ & $112-114$ & Yellow & 76 \\
\hline
\end{tabular}

Table 1: Physical characterization data of $N$ - $\alpha$-cyano- $\alpha$-phenyl methylanilines (4a-i). thiadiazolidine-1-oxide derivatives as evidenced by thin layer chromatography (TLC) showing the regioselectivity of these 1,3-dipolar cycloadditions.

The reaction afforded only one diastereomer exclusively in all cases, as evidenced by thin layer chromatography (TLC) showing the regioselectivity of these 1,3-dipolar cycloadditions (Table 2).

Structural elucidation of the 4-imino-3-phenyl-2-substitutedphenyl5-tolyl- $2 H, 3 H, 5 H[1,2,5]$ thiadiazolidine-1-oxides was unambiguously accomplished by using various spectroscopic techniques (IR, ${ }^{1} \mathrm{H}-\mathrm{NMR}$, ESI-MS) and elemental analyses data as described for compound 8a. The infrared spectrum of 4-imino-2-(4'-tolyl)-5-tolyl-3-phenyl$2 H, 3 H, 5 H[1,2,5]$ thiadiazolidine-1-oxide $8 \mathrm{a}$ exhibited absorption band<smiles>N#C[Al]=Nc1ccc([Tl])cc1</smiles>

4a, 8a: $\mathrm{R}=-\mathrm{H}$ 4b, 8b: $\mathrm{R}=-\mathrm{CH}_{3}$ 4c, 8c: $\mathrm{R}=-\mathrm{OCH}_{3}$ 4d, 8d: $\mathrm{R}=-\mathrm{Cl}$ 4e, 8 e: $\mathrm{R}=-\mathrm{NO}$ 4f, $8 \mathrm{f}: \mathrm{R}=-\mathrm{Br}$ $4 \mathrm{~g}, 8 \mathrm{~g}: \mathrm{R}=-\mathrm{OH}$

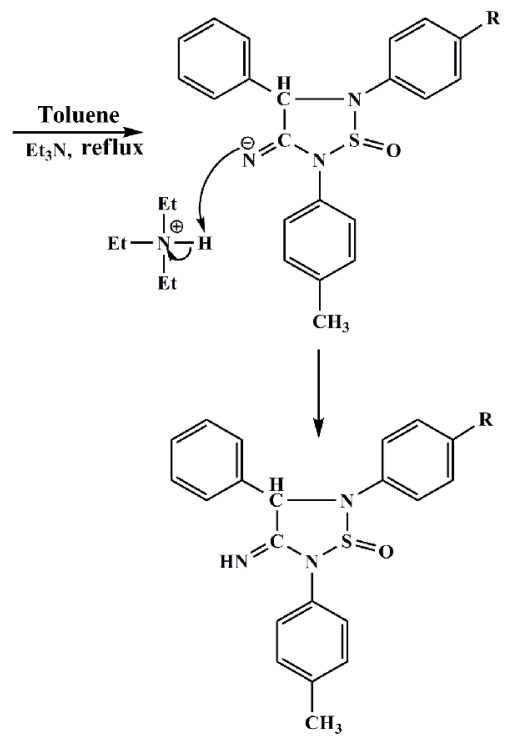

(8)
Scheme 4: Synthesis of cycloadducts and the probable mechanism.<smiles></smiles>

\begin{tabular}{|c|c|c|c|}
\hline Compounds & $\mathbf{R}$ & Melting points in ${ }^{\circ} \mathbf{C}$ & \%Yield \\
\hline $\mathbf{8 a}$ & $-\mathrm{H}$ & $122-124$ & 64 \\
\hline $\mathbf{8 b}$ & $-\mathrm{CH}_{3}$ & $116-118$ & 68 \\
\hline $\mathbf{8 c}$ & $-\mathrm{OCH}_{3}$ & $120-122$ & 62 \\
\hline $\mathbf{8 d}$ & $-\mathrm{Cl}$ & $102-104$ & 70 \\
\hline $\mathbf{8 e}$ & $-\mathrm{NO}_{2}$ & $154-156$ & 65 \\
\hline $\mathbf{8 f}$ & $-\mathrm{Br}$ & $114-116$ & 72 \\
\hline $\mathbf{8 g}$ & $-\mathrm{OH}$ & $118-120$ & 74 \\
\hline $\mathbf{8 h}$ & $-\mathrm{F}$ & $126-128$ & 69 \\
\hline $\mathbf{8 i}$ & $-\mathrm{N}\left(\mathrm{CH}_{3}\right)_{2}$ & $122-124$ & 72 \\
\hline
\end{tabular}

Table 2: Physical characterization data of 4-Imino-3-phenyl-2-substitutedphenyl-5tolyl-2H,3H,5H[1,2,5]thiadiazolidine-1-oxides (8a-i). 
Citation: Kaushal V, Kaur A, Singh B (2015) An Atom-economic and Facile Synthesis of Novel 4-Imino-3-phenyl-2-substitutedphenyl-5-tolyl$2 H, 3 H, 5 H[1,2,5]$ thiadiazolidine-1-oxide through 1,3-Dipolar Cycloaddition Reactions. Med chem 5: 484-488. doi: 10.4172/21610444.1000306

in the region of $3329 \mathrm{~cm}^{-1}$ due to $\mathrm{N}-\mathrm{H}_{\text {stretch }}$, an another band at 1616 $\mathrm{cm}^{-1}$ was assigned to $\mathrm{C}=\mathrm{N}_{\text {stretch }}$, a band at $1590 \mathrm{~cm}^{-1}$ was assigned to the skeletal stretching vibrations of the aromatic region and the absorption frequency at $1029 \mathrm{~cm}^{-1}$ has been assigned to $\mathrm{S}=\mathrm{O}_{\text {stretch }}$.

The proton magnetic resonance spectrum of $8 \mathrm{a}$ exhibited a multiplet at $\delta 6.64-8.06(14 \mathrm{H})$ that was assigned to the aromatic protons $(13 \mathrm{H})$ and amino proton $(-\mathrm{NH})$. A singlet at $\delta 5.31$ has been assigned to benzylic proton $(1 \mathrm{H})$. While the singlets at $\delta 2.33(3 \mathrm{H})$ and $\delta 2.30(3 \mathrm{H})$ have been assigned to $-\mathrm{CH}_{3}$ groups on $\mathrm{N}$-phenyl rings of azomethine and $\mathrm{N}$-sulphinyl-4-toluidine moieties respectively.

The mass spectrum of 4-imino-2-(4'-tolyl)-5-tolyl-3-phenyl$2 H, 3 H, 5 H[1,2,5]$ thiadiazolidine-1-oxide revealed the presence of the molecular ion peak at $\mathrm{m} / \mathrm{z} 375$. With loss of $N$-sulphinyl-4-toluidine from the parent ion peak a daughter ion peak at $\mathrm{m} / \mathrm{z} 222$ appears which subsequently loses a molecule of hydrocyanic acid to provide another daughter ion peak at $\mathrm{m} / \mathrm{z} 195$ via 'path a', is present which corresponds to parent azomethine and this cyclises to give ion peak at $\mathrm{m} / \mathrm{z}$ 194. This mass ion undergoes fragmentation to give mass ion at $\mathrm{m} / \mathrm{z} 107$ attributed to $p$-toluidine radical ion which may also arise by the fragmentation and rearrangement of molecular ion. Mass ion at $\mathrm{m} / \mathrm{z} 107$ may lose a hydrogen radical to give base peak at mass ion at $\mathrm{m} / \mathrm{z} 106$ forming base peak which collapses to mass ion peak at $\mathrm{m} / \mathrm{z}$ 79 which loses a molecule of ethylene to give mass ion $\mathrm{m} / \mathrm{z} 51$. The probable mode of fragmentation is as shown in the Figure 1.

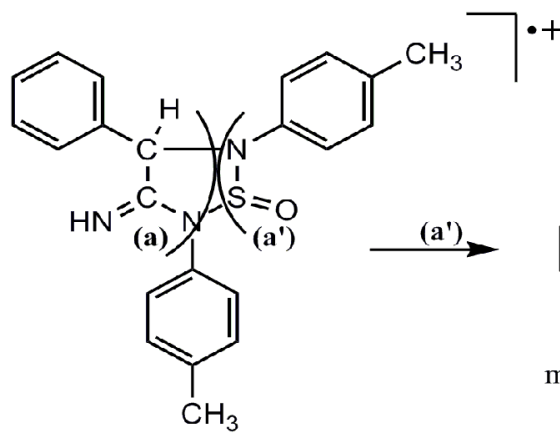

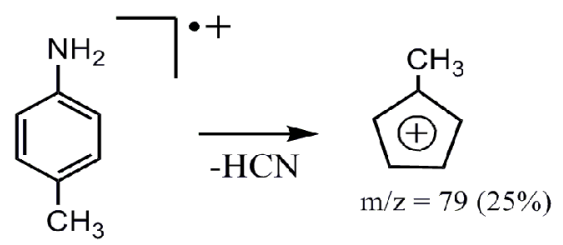

$\mathrm{m} / \mathrm{z}=375$

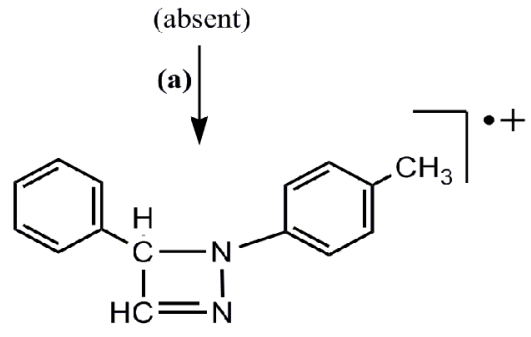<smiles>[C+]1C=CC=C1</smiles>
$\mathrm{m} / \mathrm{z}=5 \mathrm{l}(20 \%)$ $\mathrm{m} / \mathrm{z}=222(90 \%)$<smiles>Cc1ccc(N=Cc2ccccc2)cc1</smiles>
$\mathrm{m} / \mathrm{z}=195(90 \%)$<smiles></smiles>
$\mathrm{m} / \mathrm{Z}=107(8 \%)$<smiles>C=CCCC</smiles><smiles>CC(C)(C)C</smiles><smiles>Cc1ccccc1</smiles>
$\mathrm{m} / \mathrm{z}=79(25 \%)$

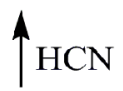<smiles>Cc1ccc(N)cc1</smiles><smiles>Cc1ccc2[nH+]cc3ccccc3c2c1</smiles><smiles>Cc1ccc(N)cc1</smiles>

$\uparrow$ $\stackrel{+}{N}$<smiles>Cc1ccc(C)cc1</smiles>

$\mathrm{m} / \mathrm{z}=194(95 \%)$ $\mathrm{m} / \mathrm{z}=107(25 \%)$

$\mathrm{m} / \mathrm{z}=106(100)$

Figure 1: The probable mass fragmentation pattern of 4-Imino-2-(4'-tolyl)-5-tolyl-3-phenyl-2H,3H,5H[1,2,5]thiadiazolidine-1-oxide 8a. 
Citation: Kaushal V, Kaur A, Singh B (2015) An Atom-economic and Facile Synthesis of Novel 4-Imino-3-phenyl-2-substitutedphenyl-5-tolyl$2 H, 3 H, 5 H[1,2,5]$ thiadiazolidine-1-oxide through 1,3-Dipolar Cycloaddition Reactions. Med chem 5: 484-488. doi: 10.4172/21610444.1000306

\section{Conclusion}

In conclusion, we have successfully developed the regioselective version of bioactive substituted thiadiazolidine derivatives through atom-economic and facile cycloaddition reactions. It was observed that the reaction took place in a stereo- and regioselective pathway across the double bond of the dipolarophiles to give novel thiadiazolidine-1oxides.

\section{Acknowledgements}

The authors acknowledge the financial support from University Grant Commission (UGC) New Delhi and are grateful to Department of Chemistry, Punjabi University, Patiala and Sophisticated Analytical Instrumentation Facility (SAIF) Panjab University, Chandigarh for spectral analysis.

\section{References}

1. Bhat AR, Tazeem, Azam A, Choi I, Athar F (2011) 3-(,3,4-Thiadiazole-2-yl) quinoline derivatives: synthesis, characterization and anti-microbial activity. Eur J Med Chem 46: 3158-3166.

2. Foroumadi A, Mansouri S, Emami S, Mirzaei J, Sorkhi M, et al. (2006) Synthesis and antibacterial activity of nitroaryl thiadiazole-levofloxacin hybrids. Arch Pharm (Weinheim) 339: 621-624.

3. Foroumadi A, Soltani F, Moshafi MH, Ashraf-Askari R (2003) Synthesis and in vitro antibacterial activity of some N-(5-aryl-,3,4-thiadiazole-2-yl)piperazinyl quinolone derivatives. Farmaco 58: 1023-1028.

4. Foroumadi A, Emami S, Hassanzadeh A, Rajaee M, Sokhanvar K, et al. (2005) Synthesis and antibacterial activity of $\mathrm{N}$-(5-benzylthio-,3,4-thiadiazol-2-yl) and $\mathrm{N}$-(5-benzylsulfonyl-,3,4-thiadiazol-2-yl)piperazinyl quinolone derivatives. Bioorg Med Chem Lett 15: 4488-4492.

5. Gadad AK, Palkar MB, Anand K, Noolvi MN, Boreddy TS, et al. (2008) Synthesis and biological evaluation of 2-trifluoromethyl/sulfonamido-5,6-diaryl substituted imidazo[2,1-b]-,3,4-thiadiazoles: a novel class of cyclooxygenase-2 inhibitors. Bioorg Med Chem 16: 276-283.

6. Gilani SJ, Khan SA, Siddiqui N (2010) Synthesis and pharmacological evaluation of condensed heterocyclic 6-substituted ,2,4-triazolo-[3,4-b]-,3,4thiadiazole and ,3,4-oxadiazole derivatives of isoniazid. Bioorg Med Chem Lett 20: $4762-4765$.

7. Al-Soud YA, Al-Masoudi NA, Loddo R, La Colla P (2008) In-vitro anti-HIV and antitumor activity of new 3,6-disubstituted [,2,4]triazolo[3,4-b][,3,4]thiadiazoles and thiadiazine analogues. Arch Pharm (Weinheim) 341: 365-369.

8. Hamilton PB, Teixeira MM, Stevens JR (2012) The evolution of Trypanosoma cruzi: the 'bat seeding' hypothesis. Trends Parasitol 28: 136-141.

9. Chen CJ, Song BA, Yang S, Xu GF, Bhadury PS, et al. (2007) Synthesis and antifungal activities of 5-(3,4,5-trimethoxyphenyl)-2-sulfonyl-,3,4-thiadiazole and 5-(3,4,5-trimethoxyphenyl)-2-sulfonyl-,3,4-oxadiazole derivatives. Bioorg Med Chem 15: 3981-3989.

10. Liu F, Luo XQ, Song BA, Bhadury PS, Yang S, et al. (2008) Synthesis and antifungal activity of novel sulfoxide derivatives containing trimethoxypheny substituted ,3,4-thiadiazole and ,3,4-oxadiazole moiety. Bioorg Med Chem 16: 3632-3640.

11. Liu XH, Shi YX, Ma Y, Zhang CY, Dong WL, et al. (2009) Synthesis, antifungal activities and 3D-QSAR study of $\mathrm{N}$-(5-substituted-,3,4-thiadiazol-2-yl) cyclopropanecarboxamides. Eur J Med Chem 44: 2782-2786.

12. Hu Y, Li CY, Wang XM, Yang YH, Zhu HL (2014) ,3,4-Thiadiazole: synthesis reactions, and applications in medicinal, agricultural, and materials chemistry. Chem Rev 114: 5572-5610.

13. Wiebe S, Jette N (2012) Pharmacoresistance and the role of surgery in difficult to treat epilepsy. Nat Rev Neurol 8: 669-677.

14. Ulloa CM, Gilliam FG (2011) Epilepsy: Improving care for patients with epilepsy. Nat Rev Neurol 7: 195-196.

15. Siddiqui N, Andalip, Bawa S, Ali R, Afzal O, et al. (2011) Antidepressant potential of nitrogen-containing heterocyclic moieties: An updated review. J Pharm Bioallied Sci 3: 194-212.

16. Singal KK, Singh B, Raj B (1999) Syn Comm 29: 911.

17. Klimenko LS, Fokin EP (1991) Photolysis of N-nitrosomethylaminoanthraquinones. Russ Chem Bull 40: 1858-1862. 